\title{
HIGHER DIMENSIONAL MINIMAL SUBMANIFOLDS GENERALIZING THE CATENOID AND HELICOID
}

\author{
JAIGyoung Choe AND Jens Hoppe
}

(Received January 6, 2012, revised May 14, 2012)

\begin{abstract}
For each $k$-dimensional complete minimal submanifold $M$ of $S^{n}$ we construct a $(k+1)$-dimensional complete minimal immersion of $M \times \boldsymbol{R}$ into $\boldsymbol{R}^{n+2}$ and $(k+1)$ dimensional minimal immersions of $M \times \boldsymbol{R}$ into $\boldsymbol{R}^{2 n+3}, \boldsymbol{H}^{2 n+3}$ and $\boldsymbol{S}^{2 n+3}$. Also from the Clifford torus $M=S^{k}(1 / \sqrt{2}) \times S^{k}(1 / \sqrt{2})$ we construct a $(2 k+2)$-dimensional complete minimal helicoid in $\boldsymbol{R}^{2 k+3}$.
\end{abstract}

Introduction. A submanifold of $\boldsymbol{R}^{n}$ whose mean curvature vector vanishes everywhere is called minimal. The mean curvature vector of a submanifold points into the direction in which the volume decreases most rapidly. Therefore minimal submanifolds are the submanifolds which are stationary with respect to volume for all compactly supported variations.

A minimal hypersurface in $\boldsymbol{R}^{n}$, viewed as a graph, satisfies the quasilinear elliptic partial differential equation:

$$
\left(1+|D u|^{2}\right) \Delta u-D_{i} u D_{j} u D_{i j} u=0 .
$$

Simons [5] showed that no entire minimal graph other than hyperplanes exists in $\boldsymbol{R}^{n}$ for $n<9$, and Bombieri, De Giorgi and Giusti [2] proved that in $\boldsymbol{R}^{n}, n \geq 9$, there exist entire minimal graphs which are not hyperplanes.

In $\boldsymbol{R}^{3}$ the catenoid and helicoid had been known to be the only two complete embedded nonplanar minimal surfaces with finite topology for two hundred years. It was in 1982 that Costa [4] surprisingly found a new complete embedded minimal surface in $\boldsymbol{R}^{3}$. From then on, tens of new complete minimal surfaces have been found in $\boldsymbol{R}^{3}$ from suitable data for the representation formula obtained by Weierstrass.

The minimal surface with the simplest Weierstrass data is the Enneper surface. It has the same total curvature of $-4 \pi$ as the catenoid and has self intersection. One quarter of the Enneper surface can be seen as a graph over a vertical half plane. By extending this property to higher dimension, the first named author [3] constructed the higher dimensional Enneper surface in $\boldsymbol{R}^{n}, 4 \leq n \leq 7$.

The catenoid is a surface of revolution in $\boldsymbol{R}^{3}$. The higher dimensional catenoid $\Sigma$ which is $S O(n-1)$-invariant minimal hypersurface in $\boldsymbol{R}^{n}$ was found by solving the ODE for the

2000 Mathematics Subject Classification. Primary 53A10; Secondary 49Q10.

Key words and phrases. Minimal submanifold, catenoid, helicoid.

Work of the first author is supported in part by R17-2008-001-01001-0. The second author would like to thank Sogang University for a research grant, and Ki-Myeong Lee for kind invitations to KIAS. Both authors thank the Swedish Research Council for support. 
generating curve of $\Sigma$ [1]. Besides the higher dimensional catenoid and the higher dimensional Enneper surface, very few examples of complete minimal submanifolds are known to exist in $\boldsymbol{R}^{n}$.

In this paper we construct many new minimal hypersurfaces and submanifolds in $\boldsymbol{R}^{n}$, and in $\boldsymbol{H}^{n}$ and $\boldsymbol{S}^{n}$ as well. All these minimal submanifolds originate from complete minimal submanifolds of $S^{n}$ just like the catenoid and helicoid arise from the minimal $S^{1}$ in $S^{2}$ and from the minimal $S^{0}$ in $S^{1}$, respectively: Given a $k$-dimensional minimal submanifold $M$ of $\boldsymbol{S}^{n}$, we can construct a $(k+1)$-dimensional complete minimal immersion of $M \times \boldsymbol{R}$ into $\boldsymbol{R}^{n+2}$. Moreover $(k+1)$-dimensional minimal immersions of $M \times \boldsymbol{R}$ are constructed in $\boldsymbol{R}^{2 n+3}$ and in $\boldsymbol{H}^{2 n+3}$ and $\boldsymbol{S}^{2 n+3}$ as well. Finally, from the Clifford torus $M=\boldsymbol{S}^{k}(1 / \sqrt{2}) \times \boldsymbol{S}^{k}(1 / \sqrt{2})$ we construct a $(2 k+2)$-dimensional complete minimal helicoid in $\boldsymbol{R}^{2 k+3}$.

1. Higher dimensional catenoid. The catenoid is a surface of revolution. The higher dimensional catenoid $\Sigma^{n-1}$ in $\boldsymbol{R}^{n}$ can be obtained from a generating curve $x=f(y)$ in $\boldsymbol{R}^{2}$ by applying the $S O(n-1)$ action. $\Sigma$ has principal vectors $v_{1}, \ldots, v_{n-1}$, where $v_{1}$ is tangent to the graph of $x=f(y)$ and $v_{2}, \ldots, v_{n-1}$ are tangent to the sphere of radius $x$. Hence the principal curvatures $\kappa_{1}, \ldots, \kappa_{n-1}$ are given by

$$
\kappa_{1}=\frac{x^{\prime \prime}}{\left\{1+\left(x^{\prime}\right)^{2}\right\}^{3 / 2}}, \quad \kappa_{2}, \ldots, \kappa_{n-1}=\frac{-1}{x \sqrt{1+\left(x^{\prime}\right)^{2}}} .
$$

Then the mean curvature of $\Sigma$ becomes

$$
H=\frac{x x^{\prime \prime}-(n-2)\left\{1+\left(x^{\prime}\right)^{2}\right\}}{x\left\{1+\left(x^{\prime}\right)^{2}\right\}^{3 / 2}} .
$$

Unlike $\Sigma^{2}$ in $\boldsymbol{R}^{3}$, the higher dimensional catenoids lie in a slab.

In this section we construct a higher dimensional minimal submanifold by interpreting the catenoid differently: The catenoid is not only a surface of revolution but also a surface spanned by a family of coaxial circles of varying radii in horizontal planes, and the circles are trivially minimal submanifolds of themselves. This observation gives the following theorem, where the coaxial circles are replaced by the minimal submanifolds $r(t) M$ of $\boldsymbol{S}^{n}(r(t))$ :

THEOREM 1. Let $M$ be a $k$-dimensional complete minimal submanifold of $\boldsymbol{S}^{n} \subset \boldsymbol{R}^{n+1}$. Suppose $r(t), t \in \boldsymbol{R}$, satisfies the second order $O D E$

$$
r \ddot{r}-k\left(1+\dot{r}^{2}\right)=0 .
$$

Then the submanifold $\Sigma \subset \boldsymbol{R}^{n+2}$ defined by $\Sigma=\{(r(t) p, t) ; p \in M, t \in \boldsymbol{R}\}$ is a complete minimal immersion of $M \times \boldsymbol{R}$ into $\boldsymbol{R}^{n+2}$.

Proof. For a fixed $p \in M$, let $C_{p}$ be the generating curve of $\Sigma$ defined by $C_{p}=$ $\{(r(t) p, t) ; t \in \boldsymbol{R}\}$. Let $x_{1}, \ldots, x_{n+2}$ be the Euclidean coordinates of $\boldsymbol{R}^{n+2}$. For a fixed $t$, $r(t) M$ denotes $\Sigma \cap\left\{x_{n+2}=t\right\}$ and $\boldsymbol{S}^{n}(r(t))=\left\{\left(x_{1}, \ldots, x_{n+1}, t\right) ; x_{1}^{2}+\cdots+x_{n+1}^{2}=r(t)^{2}\right\}$. Then one can easily find the principal vectors $v_{1}, \ldots, v_{k+1}$ of $\Sigma$ such that $v_{k+1}$ is tangent to $C_{p}$ and $v_{1}, \ldots, v_{k}$ are tangent to $r(t) M$. If $\kappa_{1}, \ldots, \kappa_{k+1}$ are the corresponding principal 
curvatures, then

$$
\kappa_{k+1}=\frac{\ddot{r}}{\left(1+\dot{r}^{2}\right)^{3 / 2}} .
$$

Since $r(t) M$ is a minimal submanifold of $S^{n}(r(t)), \sum_{i=1}^{k} \bar{\nabla}_{v_{i}} v_{i}$ is normal to $S^{n}(r(t))$ and parallel to $\left\{x_{n+2}=t\right\}$. Here $\bar{\nabla}$ denotes the connection of $\boldsymbol{R}^{n+2}$. Let $v$ be the outward unit normal to $S^{n}(r(t))$ and parallel to $\left\{x_{n+2}=t\right\}$. Then

$$
\bar{\nabla}_{v_{i}} v_{i} \cdot v=-v_{i} \cdot \bar{\nabla}_{v_{i}} v=-\frac{1}{r(t)} \text { for } i=1, \ldots, k .
$$

Hence

$$
\sum_{i=1}^{k} \bar{\nabla}_{v_{i}} v_{i}=-\frac{k}{r(t)} v
$$

and so

$$
\sum_{i=1}^{k} \kappa_{i}=-\frac{k}{r \sqrt{1+\dot{r}^{2}}} .
$$

Therefore the required ODE gives the minimality of $\Sigma$.

2. Higher dimensional helicoid. The helicoid is a ruled minimal surface. It consists of horizontal lines which are rotated as they move upward. Each horizontal line can be considered to be the cone over a minimal $S^{0}$ in $S^{1}$. The theorem of this section will replace the minimal $\boldsymbol{S}^{0}$ with the Clifford torus to construct a higher dimensional helicoid.

For the theorem we define three rigid motions:

(i) the rotation $\rho_{m}^{t}, m=1, \ldots, k+1$, of $\boldsymbol{R}^{2 k+3}$ along the $x_{m} x_{m+k+1}$-plane by

$$
\begin{aligned}
\rho_{m}^{t}\left(x_{1}, \ldots, x_{2 k+3}\right)= & \left(x_{1}, \ldots, x_{m-1}, x_{m} \cos t-x_{m+k+1} \sin t, x_{m+1}, \ldots,\right. \\
& \left.x_{m+k}, x_{m} \sin t+x_{m+k+1} \cos t, x_{m+k+2}, \ldots, x_{2 k+3}\right) ;
\end{aligned}
$$

(ii) the translation $\tau_{t}$ by

$$
\tau_{t}\left(x_{1}, \ldots, x_{2 k+2}, x_{2 k+3}\right)=\left(x_{1}, \ldots, x_{2 k+2}, x_{2 k+3}+t\right) ;
$$

(iii) the multi-screw motion $\sigma_{t}$ by

$$
\sigma_{t}=\rho_{1}^{t} \circ \cdots \circ \rho_{k+1}^{t} \circ \tau_{t} .
$$

The rotation by $90^{\circ}$ in $\boldsymbol{R}^{2}$ is represented by the matrix $J=\left(\begin{array}{cc}0 & -1 \\ 1 & 0\end{array}\right)$. Let $I$ be the $(k+$ $1) \times(k+1)$ identity matrix and define a $(2 k+2) \times(2 k+2)$ matrix $\boldsymbol{J}=\left(\begin{array}{cc}0 & -I \\ I & 0\end{array}\right)$ written in block matrices. Then the multi-screw motion can be simply represented by

$$
\sigma_{t}\left(\mathbf{x}, x_{2 k+3}\right)=\left(e^{\boldsymbol{J} t} \mathbf{x}, x_{2 k+3}+t\right), \quad \mathbf{x} \in \boldsymbol{R}^{2 k+2} .
$$

Given a skew symmetric matrix $A, A^{T}=-A$, we will use the following facts in the proof.

$$
\left(e^{A t}\right)^{T}=e^{-A t}=\left(e^{A t}\right)^{-1}, \quad \frac{d}{d t} e^{A t}=A e^{A t}, \quad A e^{A T}=e^{A T} A .
$$


THEOREM 2. Let $M$ be the Clifford torus $\boldsymbol{S}^{k}(1 / \sqrt{2}) \times \boldsymbol{S}^{k}(1 / \sqrt{2})$ in $\boldsymbol{S}^{2 k+1} \subset \boldsymbol{R}^{2 k+2}$. Assuming that $M$ lies in the hyperplane $\left\{x_{2 k+3}=0\right\}$ of $\boldsymbol{R}^{2 k+3}$, define

$$
\Sigma=\bigcup_{t \in \boldsymbol{R}} \sigma_{t}(O \circledast M)
$$

where $O$ is the origin of $\boldsymbol{R}^{2 k+2}$ and $O \circledast M$ is the infinite cone over $M$, i.e., the union of the rays from $O$ over the points of $M$. Then $\Sigma$ is a $(2 k+2)$-dimensional complete minimal hypersurface in $\boldsymbol{R}^{2 k+3}$. $\Sigma$ has a 1-dimensional singular set $\{(0, \ldots, 0, t) ; t \in \boldsymbol{R}\}$.

Proof. $O \circledast M$ has a singular set $\{O\}$. Since $\sigma_{a}(\Sigma)=\Sigma$, the singular set of $\Sigma$ is the $x_{2 k+3}$-axis.

Let $\varphi_{1}, \ldots, \varphi_{2 k}$ be local coordinates on $M$ such that $\mathbf{m}: D \subset \boldsymbol{R}^{2 k} \rightarrow M$ is a local immersion defined as

$$
\mathbf{m}\left(\varphi_{1}, \ldots, \varphi_{2 k}\right)=\frac{1}{\sqrt{2}}\left(\begin{array}{c}
\mathbf{m}_{1}\left(\varphi_{1}, \ldots, \varphi_{k}\right) \\
\mathbf{m}_{2}\left(\varphi_{k+1}, \ldots, \varphi_{2 k}\right)
\end{array}\right),
$$

where $\mathbf{m}_{1}, \mathbf{m}_{2}$ are immersions on $\boldsymbol{S}^{k}$. Define a local immersion $\boldsymbol{X}$ for $\Sigma$ by

$$
\boldsymbol{X}\left(\varphi_{1}, \ldots, \varphi_{2 k}, t, r\right)=\left(\begin{array}{c}
r e^{\boldsymbol{J} t} \mathbf{m}\left(\varphi_{1}, \ldots, \varphi_{2 k}\right) \\
t
\end{array}\right) .
$$

The metrics of $M$ and $\Sigma$ are

$$
d s_{M}^{2}=\sum_{a, b=1}^{2 k} \bar{g}_{a b} d \varphi_{a} d \varphi_{b} \text { and } d s_{\Sigma}^{2}=\sum_{i, j=1}^{2 k+2} G_{i j} d \varphi_{i} d \varphi_{j},
$$

where $\varphi_{2 k+1}:=t, \varphi_{2 k+2}:=r$.

Concerning $\left(G_{i j}\right)$ one computes

$$
\begin{aligned}
G_{a b} & =\frac{\partial \boldsymbol{X}}{\partial \varphi_{a}} \cdot \frac{\partial \boldsymbol{X}}{\partial \varphi_{b}}=\left(\begin{array}{c}
r e^{\boldsymbol{J} t} \frac{\partial \mathbf{m}}{\partial \varphi_{a}} \\
0
\end{array}\right) \cdot\left(\begin{array}{c}
r e^{\boldsymbol{J} t} \frac{\partial \mathbf{m}}{\partial \varphi_{b}} \\
0
\end{array}\right)=r^{2} \bar{g}_{a b}, \\
G_{a 2 k+1} & =\frac{\partial \boldsymbol{X}}{\partial \varphi_{a}} \cdot \frac{\partial \boldsymbol{X}}{\partial t}=\left(\begin{array}{c}
r e^{\boldsymbol{J} t} \frac{\partial \mathbf{m}}{\partial \varphi_{a}} \\
0
\end{array}\right) \cdot\left(\begin{array}{c}
r \boldsymbol{J} e^{\boldsymbol{J} t} \mathbf{m} \\
1
\end{array}\right) \\
& =r^{2} \frac{\partial \mathbf{m}}{\partial \varphi_{a}} \cdot \boldsymbol{J} \mathbf{m}=\frac{r^{2}}{2}\left(\mathbf{m}_{1} \cdot \frac{\partial \mathbf{m}_{2}}{\partial \varphi_{a}}-\mathbf{m}_{2} \cdot \frac{\partial \mathbf{m}_{\mathbf{1}}}{\partial \varphi_{a}}\right):=r^{2} \bar{z}_{a}, \\
G_{a 2 k+2} & =\frac{\partial \boldsymbol{X}}{\partial \varphi_{a}} \cdot \frac{\partial \boldsymbol{X}}{\partial r}=\left(\begin{array}{c}
r e^{\boldsymbol{J} t} \frac{\partial \mathbf{m}}{\partial \varphi_{a}} \\
0
\end{array}\right) \cdot\left(\begin{array}{c}
e^{\boldsymbol{J} t} \mathbf{m} \\
0
\end{array}\right)=0, \\
G_{2 k+12 k+1} & =\frac{\partial \boldsymbol{X}}{\partial t} \cdot \frac{\partial \boldsymbol{X}}{\partial t}=\left(\begin{array}{c}
r \boldsymbol{J} e^{\boldsymbol{J} t} \mathbf{m} \\
1
\end{array}\right) \cdot\left(\begin{array}{c}
r \boldsymbol{J} e^{\boldsymbol{J} t} \mathbf{m} \\
1
\end{array}\right)=r^{2}+1, \\
G_{2 k+12 k+2} & =\frac{\partial \boldsymbol{X}}{\partial t} \cdot \frac{\partial \boldsymbol{X}}{\partial r}=\left(\begin{array}{c}
r \boldsymbol{J} e^{\boldsymbol{J} t} \mathbf{m} \\
1
\end{array}\right) \cdot\left(\begin{array}{c}
e^{\boldsymbol{J} t} \mathbf{m} \\
0
\end{array}\right)=0, \\
G_{2 k+22 k+2} & =\frac{\partial \boldsymbol{X}}{\partial r} \cdot \frac{\partial \boldsymbol{X}}{\partial r}=\left(\begin{array}{c}
e^{\boldsymbol{J} t} \mathbf{m} \\
0
\end{array}\right) \cdot\left(\begin{array}{c}
e^{\boldsymbol{J} t} \mathbf{m} \\
0
\end{array}\right)=1 .
\end{aligned}
$$


Therefore

$$
\left(G_{i j}\right)=r^{2}\left(\begin{array}{ccccc}
\bar{g}_{11} & \cdots & \bar{g}_{12 k} & \bar{z}_{1} & 0 \\
\cdot & \cdots & \cdot & \cdot & \cdot \\
\cdot & \cdots & \cdot & \cdot & \cdot \\
\bar{g}_{2 k 1} & \cdots & \bar{g}_{2 k 2 k} & \bar{z}_{2 k} & 0 \\
\bar{z}_{1} & \cdots & \bar{z}_{2 k} & \left(r^{2}+1\right) / r^{2} & 0 \\
0 & \cdots & 0 & 0 & 1 / r^{2}
\end{array}\right)
$$

where

$$
\bar{z}_{a}=\frac{1}{2}\left(\mathbf{m}_{1} \cdot \frac{\partial \mathbf{m}_{2}}{\partial \varphi_{a}}-\mathbf{m}_{2} \cdot \frac{\partial \mathbf{m}_{\mathbf{1}}}{\partial \varphi_{a}}\right)
$$

and

$$
\left(\bar{g}_{a b}\right)=\left(\begin{array}{cccccc}
\bar{g}_{11} & \cdots & \bar{g}_{1 k} & 0 & \cdots & 0 \\
\cdot & \cdots & \cdot & \cdot & \cdots & \cdot \\
\cdot & \cdots & \cdot & \cdot & \cdots & \cdot \\
\bar{g}_{k 1} & \cdots & \bar{g}_{k k} & 0 & \cdots & 0 \\
0 & \cdots & 0 & \bar{g}_{k+1 k+1} & \cdots & \bar{g}_{k+12 k} \\
\cdot & \cdots & \cdot & \cdot & \cdots & \cdot \\
\cdot & \cdots & \cdot & \cdot & \cdots & \cdot \\
0 & \cdots & 0 & \bar{g}_{2 k k+1} & \cdots & \bar{g}_{2 k 2 k}
\end{array}\right) .
$$

It is not difficult to verify that the inverse of $\left(G_{i j}\right)$ is

$$
\left(G^{i j}\right)=\frac{1}{r^{2}}\left(\begin{array}{ccccc}
\bar{g}^{11} & \cdots & \bar{g}^{12 k} & 0 & 0 \\
\cdot & \cdots & \cdot & \cdot & \cdot \\
\cdot & \cdots & \cdot & \cdot & \cdot \\
\bar{g}^{2 k} 1 & \cdots & \bar{g}^{2 k} 2 k & 0 & 0 \\
0 & \cdots & 0 & 0 & 0 \\
0 & \cdots & 0 & 0 & r^{2}
\end{array}\right)+\frac{1}{r^{2} Z}\left(\begin{array}{ccccc}
\bar{z}^{1} \bar{z}^{1} & \cdots & \bar{z}^{1} \bar{z}^{2 k} & -\bar{z}^{1} & 0 \\
\cdot & \cdots & \cdot & \cdot & \cdot \\
\cdot & \cdots & \cdot & \cdot & \cdot \\
\bar{z}^{2 k} \bar{z}^{1} & \cdots & \left(\bar{z}^{2 k}\right)^{2} & -\bar{z}^{2 k} & 0 \\
-\bar{z}^{1} & \cdots & -\bar{z}^{2 k} & 1 & 0 \\
0 & \cdots & 0 & 0 & 0
\end{array}\right),
$$

where

$$
Z=\left(1+\frac{1}{r^{2}}-\sum_{a} \bar{z}_{a} \bar{z}^{a}\right), \quad \bar{z}^{a}=\sum_{b} \bar{g}^{a b} \bar{z}_{b} .
$$

Now we need to compute the second fundamental form of $M$. Since $M=\left\{x_{1}^{2}+\cdots+\right.$ $\left.x_{k+1}^{2}-\left(x_{k+2}^{2}+\cdots+x_{2 k+2}^{2}\right)=0\right\} \cap S^{2 k+1}$, the unit normal $\mathbf{n}$ to $M$ in $S^{2 k+1}$ is given by

$$
\mathbf{n}=\frac{1}{\sqrt{2}}\left(\begin{array}{c}
\mathbf{m}_{1} \\
-\mathbf{m}_{2}
\end{array}\right) .
$$

Then a normal $v$ to $\Sigma$ in $\boldsymbol{R}^{2 k+3}$ should be of the form

$$
v=\left(\begin{array}{c}
e^{J t} \mathbf{n} \\
d r
\end{array}\right)
$$


for some constant $d$. From $v \perp \partial \boldsymbol{X} / \partial t$ we get $d=\mathbf{m}_{1} \cdot \mathbf{m}_{2}$. Denote by $\left(\bar{h}_{a b}\right)$ the second fundamental form of $M$ in $S^{2 k+1}$ with respect to $\varphi_{1}, \ldots, \varphi_{2 k}$ and $\mathbf{n}$, that is,

$$
\bar{h}_{a b}=\frac{\partial^{2} \mathbf{m}}{\partial \varphi_{a} \partial \varphi_{b}} \cdot \mathbf{n} .
$$

And let $\left(H_{i j}\right)$ be the second fundamental form of $\Sigma$ in $\boldsymbol{R}^{2 k+3}$ with respect to $\varphi_{1}, \ldots, \varphi_{2 k}, t, r$ and $v /|v|$. Then

$$
\begin{aligned}
& H_{a b}=\frac{\partial^{2} \boldsymbol{X}}{\partial \varphi_{a} \partial \varphi_{b}} \cdot \frac{\nu}{|\nu|}=\frac{1}{|\nu|}\left(\begin{array}{c}
r e^{J t} \frac{\partial^{2} \mathbf{m}}{\partial \varphi_{a} \partial \varphi_{b}} \\
0
\end{array}\right) \cdot\left(\begin{array}{c}
e^{J t} \mathbf{n} \\
d r
\end{array}\right)=\frac{r}{|\nu|} \bar{h}_{a b}, \\
& H_{a 2 k+1}=\frac{\partial^{2} \boldsymbol{X}}{\partial \varphi_{a} \partial t} \cdot \frac{\nu}{|\nu|}=\frac{1}{|\nu|}\left(\begin{array}{c}
r \boldsymbol{J} e^{\boldsymbol{J} t} \frac{\partial \mathbf{m}}{\partial \varphi_{a}} \\
0
\end{array}\right) \cdot\left(\begin{array}{c}
e^{\boldsymbol{J} t} \mathbf{n} \\
d r
\end{array}\right) \\
& =-\frac{r}{2|\nu|}\left(\mathbf{m}_{1} \cdot \frac{\partial \mathbf{m}_{2}}{\partial \varphi_{a}}+\mathbf{m}_{2} \cdot \frac{\partial \mathbf{m}_{1}}{\partial \varphi_{a}}\right) \text {, } \\
& H_{a 2 k+2}=\frac{\partial^{2} \boldsymbol{X}}{\partial \varphi_{a} \partial r} \cdot \frac{v}{|v|}=\frac{1}{|v|}\left(\begin{array}{c}
e^{J t} \frac{\partial \mathbf{m}}{\partial \varphi_{a}} \\
0
\end{array}\right) \cdot\left(\begin{array}{c}
e^{\boldsymbol{J} t} \mathbf{n} \\
d r
\end{array}\right)=0, \\
& H_{2 k+12 k+1}=\frac{\partial^{2} \boldsymbol{X}}{\partial^{2} t} \cdot \frac{v}{|v|}=\frac{1}{|\nu|}\left(\begin{array}{c}
r \boldsymbol{J}^{2} e^{J t} \mathbf{m} \\
0
\end{array}\right) \cdot\left(\begin{array}{c}
e^{\boldsymbol{J} t} \mathbf{n} \\
d r
\end{array}\right)=0, \\
& H_{2 k+12 k+2}=\frac{\partial^{2} \boldsymbol{X}}{\partial t \partial r} \cdot \frac{\nu}{|\nu|}=\frac{1}{|\nu|}\left(\begin{array}{c}
\boldsymbol{J} e^{\boldsymbol{J} t} \mathbf{m} \\
0
\end{array}\right) \cdot\left(\begin{array}{c}
e^{\boldsymbol{J} t} \mathbf{n} \\
d r
\end{array}\right)=\frac{1}{|\nu|} \mathbf{m} \cdot(-\boldsymbol{J}) \mathbf{n} \\
& =-\frac{\mathbf{m}_{1} \cdot \mathbf{m}_{2}}{|v|} \text {, } \\
& H_{2 k+22 k+2}=\frac{\partial^{2} \boldsymbol{X}}{\partial^{2} r} \cdot \frac{\nu}{|\nu|}=\frac{1}{|\nu|}\left(\begin{array}{l}
0 \\
0
\end{array}\right) \cdot\left(\begin{array}{c}
e^{J t} \mathbf{n} \\
d r
\end{array}\right)=0 .
\end{aligned}
$$

Therefore

$$
\left(H_{i j}\right)=\frac{r}{|v|}\left(\begin{array}{ccccc}
\bar{h}_{11} & \cdots & \bar{h}_{12 k} & \bar{y}^{1} & 0 \\
\cdot & \cdots & \cdot & \cdot & . \\
\cdot & \cdots & . & . & . \\
\bar{h}_{2 k 1} & \cdots & \bar{h}_{2 k} 2 k & \bar{y}^{2 k} & 0 \\
\bar{y}^{1} & \cdots & \bar{y}^{2 k} & 0 & -\frac{1}{r} \mathbf{m}_{1} \cdot \mathbf{m}_{2} \\
0 & \cdots & 0 & -\frac{1}{r} \mathbf{m}_{1} \cdot \mathbf{m}_{2} & 0
\end{array}\right)
$$

where

$$
\bar{y}^{a}:=-\frac{1}{2}\left(\mathbf{m}_{1} \cdot \frac{\partial \mathbf{m}_{2}}{\partial \varphi_{a}}+\mathbf{m}_{2} \cdot \frac{\partial \mathbf{m}_{1}}{\partial \varphi_{a}}\right) .
$$

But it should be remarked that since

$$
\bar{h}_{a b}=\frac{\partial^{2} \mathbf{m}}{\partial \varphi_{a} \partial \varphi_{b}} \cdot \mathbf{n}=-\frac{\partial \mathbf{m}}{\partial \varphi_{a}} \cdot \frac{\partial \mathbf{n}}{\partial \varphi_{b}}=\frac{1}{2}\left(-\frac{\partial \mathbf{m}_{1}}{\partial \varphi_{a}} \cdot \frac{\partial \mathbf{m}_{1}}{\partial \varphi_{b}}+\frac{\partial \mathbf{m}_{2}}{\partial \varphi_{a}} \cdot \frac{\partial \mathbf{m}_{2}}{\partial \varphi_{b}}\right)
$$


we have

$$
\left(\bar{h}_{a b}\right)=\left(\begin{array}{cccccc}
-\bar{g}_{11} & \cdots & -\bar{g}_{1 k} & 0 & \cdots & 0 \\
\cdot & \cdots & \cdot & \cdot & \cdots & \cdot \\
\cdot & \cdots & \cdot & \cdot & \cdots & \cdot \\
-\bar{g}_{k 1} & \cdots & -\bar{g}_{k k} & 0 & \cdots & 0 \\
0 & \cdots & 0 & \bar{g}_{k+1 k+1} & \cdots & \bar{g}_{k+12 k} \\
\cdot & \cdots & \cdot & \cdot & \cdots & \cdot \\
\cdot & \cdots & \cdot & \cdot & \cdots & \cdot \\
0 & \cdots & 0 & \bar{g}_{2 k k+1} & \cdots & \bar{g}_{2 k 2 k}
\end{array}\right) .
$$

Finally we can compute the mean curvature $H$ of $\Sigma$ in $\boldsymbol{R}^{2 k+3}$ as follows.

$$
\begin{aligned}
H=\sum_{i, j} G^{i j} H_{i j}= & \frac{1}{r|v|}\left(\sum_{a, b} \bar{g}^{a b} \bar{h}_{a b}\right)+\frac{r}{|\nu| Z} \sum_{a, b} \bar{h}_{a b} \bar{z}^{a} \bar{z}^{b} \\
& +\frac{r}{|\nu| Z} \sum_{a} \bar{z}^{a}\left(\mathbf{m}_{1} \cdot \frac{\partial \mathbf{m}_{2}}{\partial \varphi_{a}}+\mathbf{m}_{2} \cdot \frac{\partial \mathbf{m}_{1}}{\partial \varphi_{a}}\right) \\
:= & H_{0}+H_{1}+H_{2} .
\end{aligned}
$$

Clearly $H_{0}=0$ since $M$ is minimal in $S^{2 k+1}$. On the other hand,

$$
\begin{gathered}
H_{2}=\frac{r}{|\nu| Z} \sum_{a, b} \bar{g}^{a b} \frac{1}{2}\left(\mathbf{m}_{1} \cdot \frac{\partial \mathbf{m}_{2}}{\partial \varphi_{b}}-\mathbf{m}_{2} \cdot \frac{\partial \mathbf{m}_{1}}{\partial \varphi_{b}}\right)\left(\mathbf{m}_{1} \cdot \frac{\partial \mathbf{m}_{2}}{\partial \varphi_{a}}+\mathbf{m}_{2} \cdot \frac{\partial \mathbf{m}_{1}}{\partial \varphi_{a}}\right) \\
=\frac{r}{2|\nu| Z} \sum_{a, b} \bar{g}^{a b}\left(\mathbf{m}_{1} \cdot \frac{\partial \mathbf{m}_{2}}{\partial \varphi_{b}} \mathbf{m}_{1} \cdot \frac{\partial \mathbf{m}_{2}}{\partial \varphi_{a}}-\mathbf{m}_{2} \cdot \frac{\partial \mathbf{m}_{1}}{\partial \varphi_{b}} \mathbf{m}_{2} \cdot \frac{\partial \mathbf{m}_{1}}{\partial \varphi_{a}}\right), \\
H_{1}=\frac{r}{4|\nu| Z} \sum_{a, b, c, d}\left(\mp \bar{g}_{a b}\right) \bar{g}^{a c}\left(\mathbf{m}_{1} \cdot \frac{\partial \mathbf{m}_{2}}{\partial \varphi_{c}}-\mathbf{m}_{2} \cdot \frac{\partial \mathbf{m}_{1}}{\partial \varphi_{c}}\right) \bar{g}^{b d}\left(\mathbf{m}_{1} \cdot \frac{\partial \mathbf{m}_{2}}{\partial \varphi_{d}}-\mathbf{m}_{2} \cdot \frac{\partial \mathbf{m}_{1}}{\partial \varphi_{d}}\right),
\end{gathered}
$$

where the negative sign in $\mp \bar{g}_{a b}$ occurs if $1 \leq a \leq k$ and the positive sign if $k+1 \leq a \leq 2 k$.

Hence by the block-diagonality of $\bar{g}_{a b}$

$$
H_{1}=\frac{r}{4|\nu| Z} \sum_{c, d} \bar{g}^{c d}\left(\mathbf{m}_{1} \cdot \frac{\partial \mathbf{m}_{2}}{\partial \varphi_{c}} \mathbf{m}_{1} \cdot \frac{\partial \mathbf{m}_{2}}{\partial \varphi_{d}}-\mathbf{m}_{2} \cdot \frac{\partial \mathbf{m}_{1}}{\partial \varphi_{c}} \mathbf{m}_{2} \cdot \frac{\partial \mathbf{m}_{1}}{\partial \varphi_{d}}\right) .
$$

Therefore the theorem will follow from the following lemma.

LEMMA.

$$
\frac{1}{2} \sum_{a, b} \bar{g}^{a b} \mathbf{m}_{1} \cdot \frac{\partial \mathbf{m}_{2}}{\partial \varphi_{a}} \mathbf{m}_{1} \cdot \frac{\partial \mathbf{m}_{2}}{\partial \varphi_{b}}=\frac{1}{2} \sum_{a, b} \bar{g}^{a b} \mathbf{m}_{2} \cdot \frac{\partial \mathbf{m}_{1}}{\partial \varphi_{a}} \mathbf{m}_{2} \cdot \frac{\partial \mathbf{m}_{1}}{\partial \varphi_{b}}=1-\left(\mathbf{m}_{1} \cdot \mathbf{m}_{2}\right)^{2} .
$$

Proof of Lemma. $k+1$ vectors $\mathbf{m}_{1}, \partial \mathbf{m}_{1} / \partial \varphi_{1}, \ldots, \partial \mathbf{m}_{1} / \partial \varphi_{k}$ form a basis for $\boldsymbol{R}^{k+1} \supset \boldsymbol{S}^{k}$. Hence

$$
\mathbf{m}_{2}=\left(\mathbf{m}_{2} \cdot \mathbf{m}_{1}\right) \mathbf{m}_{1}+\frac{1}{2} \sum_{a, b} \bar{g}^{a b}\left(\mathbf{m}_{2} \cdot \frac{\partial \mathbf{m}_{1}}{\partial \varphi_{a}}\right) \frac{\partial \mathbf{m}_{1}}{\partial \varphi_{b}} .
$$


Then

$$
1=\left|\mathbf{m}_{2}\right|^{2}=\left(\mathbf{m}_{1} \cdot \mathbf{m}_{2}\right)^{2}+\frac{1}{2} \sum_{a, b} \bar{g}^{a b} \mathbf{m}_{2} \cdot \frac{\partial \mathbf{m}_{1}}{\partial \varphi_{a}} \mathbf{m}_{2} \cdot \frac{\partial \mathbf{m}_{1}}{\partial \varphi_{b}} .
$$

Interchanging the roles of $\mathbf{m}_{1}$ and $\mathbf{m}_{2}$ yields the proof.

3. Higher codimension. We have seen in Section 1 that if one expands a minimal submanifold $M$ of $\boldsymbol{S}^{n}$ into $r(t) M \subset S^{n}(r(t))$ as time $t$ changes, with $r(t)$ as in Theorem 1, then the set $\Sigma$ swept out by $r(t) M$ is the higher dimensional catenoid. The minimality of $\Sigma$ results from the fact that the principal curvatures arising from the submanifold $M$ of $\boldsymbol{R}^{n+1}$ are balanced by the principal curvature of the generating curve $C_{p}=\{(r(t) p, t) ; p \in M, t \in \boldsymbol{R}\}$. In other words, the expansion of $M$ gives a balancing curvature to $M$. We have another natural method, the rotation, which can also create a balancing curvature through the centrifugal force. In this section we will combine an expansion by a factor of $r(t)$ and a rotation by an angle of $\theta(t)$ : we will rotate $r(t) M$ from one $\boldsymbol{R}^{n+1}$ to another independent $\boldsymbol{R}^{n+1}$ in $\boldsymbol{R}^{2 n+3}$ to construct a minimal immersion of $M \times \boldsymbol{R}$ into $\boldsymbol{R}^{2 n+3}$.

THEOREM 3. Let $M$ be a $k$-dimensional complete minimal submanifold of $\boldsymbol{S}^{n}$ locally defined by the immersion $\mathbf{m}\left(\varphi_{1}, \ldots, \varphi_{k}\right)$. Define $\boldsymbol{X}: M \times \boldsymbol{R} \rightarrow \boldsymbol{R}^{2 n+3}$ by

$$
\boldsymbol{X}\left(\varphi_{1}, \ldots, \varphi_{k}, t\right)=\left(\begin{array}{c}
r(t) \cos \theta(t) \mathbf{m}\left(\varphi_{1}, \ldots, \varphi_{k}\right) \\
r(t) \sin \theta(t) \mathbf{m}\left(\varphi_{1}, \ldots, \varphi_{k}\right) \\
t
\end{array}\right) .
$$

Then $\boldsymbol{X}$ is a complete minimal immersion, provided $r(t)$ and $\theta(t)$ satisfy

$$
\dot{r}^{2}+\frac{L^{2}}{r^{2}}-\left(\frac{r}{r_{0}}\right)^{2 k}=-1
$$

and

$$
r^{2} \dot{\theta}=L
$$

where $r_{0}$ and the angular momentum $L$ are constants.

PROOF. Let $d s_{M}^{2}=\sum_{a, b=1}^{k} \bar{g}_{a b} d \varphi_{a} d \varphi_{b}$ be the metric of $M$ such that $\bar{g}_{a b}=\partial \mathbf{m} / \partial \varphi_{a}$. $\partial \mathbf{m} / \partial \varphi_{b}$ and $\bar{g}=\operatorname{det}\left(\bar{g}_{a b}\right)$. If $\Sigma$ denotes the image of the immersion $\boldsymbol{X}$, then the metric of $\Sigma$ can be written as

$$
d s_{\Sigma}^{2}=\sum_{a, b=1}^{k} g_{a b} d \varphi_{a} d \varphi_{b}+g_{k+1 k+1} d t^{2}
$$

such that

$$
g_{a b}=r^{2} \bar{g}_{a b}, \quad g_{k+1 k+1}=|\dot{\boldsymbol{X}}|^{2} .
$$


Here we used the property that $\dot{\boldsymbol{X}} \cdot \partial \boldsymbol{X} / \partial \varphi_{a}=0$. If $\sqrt{g} d \varphi_{1} \cdots d \varphi_{k} d t$ denotes the volume form of $\Sigma$, then $g=r^{2 k}|\dot{X}|^{2} \bar{g}$. Now

$$
\dot{\boldsymbol{X}}=\left(\begin{array}{c}
(\dot{r} \cos \theta-r \dot{\theta} \sin \theta) \mathbf{m} \\
(\dot{r} \sin \theta+r \dot{\theta} \cos \theta) \mathbf{m} \\
1
\end{array}\right)
$$

hence by (3) and (2)

$$
|\dot{\boldsymbol{X}}|^{2}=\dot{r}^{2}+r^{2} \dot{\theta}^{2}+1=\dot{r}^{2}+\frac{L^{2}}{r^{2}}+1=\left(\frac{r}{r_{0}}\right)^{2 k}
$$

and

$$
g=\frac{r^{4 k}}{\left(r_{0}\right)^{2 k}} \bar{g} .
$$

Differentiating (2) and (3) with respect to $t$ yields

$$
\ddot{r}-\frac{L^{2}}{r^{3}}=\frac{k r^{2 k-1}}{\left(r_{0}\right)^{2 k}},
$$

and

$$
2 \dot{r} \dot{\theta}+r \ddot{\theta}=0
$$

respectively. Recall that given a smooth function $f$ on a Riemannian manifold $\left(N, d s_{N}^{2}=\right.$ $\left.\sum G_{A B} d \psi_{A} d \psi_{B}\right)$ with $G=\operatorname{det}\left(G_{A B}\right)$, we have

$$
\Delta_{N} f=\frac{1}{\sqrt{G}} \sum \frac{\partial}{\partial \psi_{A}}\left(\sqrt{G} G^{A B} \frac{\partial}{\partial \psi_{B}} f\right) .
$$

To prove that $\boldsymbol{X}$ is a minimal immersion, it suffices to show $\Delta_{\Sigma} x_{i}=0$, where $\boldsymbol{X}\left(\varphi_{1}, \ldots\right.$, $\left.\varphi_{k}, t\right)=\left(x_{1}, \ldots, x_{2 n+3}\right)^{T}$. First

$$
\begin{aligned}
\Delta_{\Sigma} x_{2 n+3} & =\Delta_{\Sigma} t=\frac{1}{\sqrt{g}} \frac{\partial}{\partial t}\left(\sqrt{g} g^{k+1 k+1}\right) \\
& =\left(\frac{\left(r_{0}\right)^{k}}{r^{2 k} \sqrt{\bar{g}}}\right) \frac{\partial}{\partial t}\left(\left(r_{0}\right)^{k} \sqrt{\bar{g}}\right) \\
& =0 .
\end{aligned}
$$

Second, for $i=1, \ldots, n+1$, assuming $\mathbf{m}^{T}=\left(m_{1}, \ldots, m_{n+1}\right)$, we have

$$
\begin{aligned}
& \Delta_{\Sigma} x_{i}=\Delta_{\Sigma}\left(r \cos \theta m_{i}\right)=\frac{\left(r_{0}\right)^{k}}{r^{2 k} \sqrt{g}} \sum_{a, b=1}^{k} \frac{\partial}{\partial \varphi_{a}}\left\{\frac{r^{2 k} \sqrt{g}}{\left(r_{0}\right)^{k}} \frac{\bar{g}^{a b}}{r^{2}} \frac{\partial}{\partial \varphi_{b}}\left(r \cos \theta m_{i}\right)\right\} \\
& +\frac{\left(r_{0}\right)^{k}}{r^{2 k} \sqrt{g}} \frac{\partial}{\partial t}\left\{\frac{r^{2 k} \sqrt{g}}{\left(r_{0}\right)^{k}}\left(\frac{r_{0}}{r}\right)^{2 k} \frac{\partial}{\partial t}\left(r \cos \theta m_{i}\right)\right\} \\
& =\frac{\cos \theta}{r \sqrt{\bar{g}}} \sum_{a, b=1}^{k} \frac{\partial}{\partial \varphi_{a}}\left(\sqrt{\bar{g}} \bar{g}^{a b} \frac{\partial}{\partial \varphi_{b}} m_{i}\right)+\left(\frac{r_{0}}{r}\right)^{2 k}\left(\frac{\partial^{2}}{\partial t^{2}}(r \cos \theta)\right) m_{i}
\end{aligned}
$$




$$
\begin{aligned}
= & -\frac{k \cos \theta}{r} m_{i}+\left(\frac{r_{0}}{r}\right)^{2 k}\left(\ddot{r} \cos \theta-2 \dot{r} \dot{\theta} \sin \theta-r \ddot{\theta} \sin \theta-r \dot{\theta}^{2} \cos \theta\right) m_{i} \\
& \left.\quad \text { because } \Delta_{M} \mathbf{m}+k \mathbf{m}=\mathbf{0}\right) \\
= & -\frac{k \cos \theta}{r} m_{i}+\left(\frac{r_{0}}{r}\right)^{2 k}\left(\ddot{r}-\frac{L^{2}}{r^{3}}\right) \cos \theta m_{i} \quad \text { (by (5) and (3)) } \\
= & 0 . \quad(\text { by (4)) }
\end{aligned}
$$

Similarly for $x_{i+n+1}=r \sin \theta m_{i}, i=1, \ldots, n+1$, we have

$$
\Delta_{\Sigma} x_{i+n+1}=0 \text {. }
$$

REMARK. When $L=0$ in Theorem 3, $M$ is just the higher dimensional catenoid of Theorem 1.

4. Non-Euclidean spaces. The minimal immersion of $M$ in $S^{n}$ can be extended to the minimal immersion of $M \times \boldsymbol{R}$ in $\boldsymbol{H}^{2 n+3}$ and in $\boldsymbol{S}^{2 k+3}$ as well as in $\boldsymbol{R}^{2 n+3}$.

THEOREM 4. Let $M$ be a $k$-dimensional complete minimal submanifold of $\boldsymbol{S}^{n}$ locally defined by the immersion $\mathbf{m}\left(\varphi_{1}, \ldots, \varphi_{k}\right)$. Also let $\boldsymbol{H}^{2 n+3}=\left\{x_{1}^{2}+\cdots+x_{2 n+3}^{2}-x_{2 n+4}^{2}=-1\right\}$ be the hyperbolic space embedded in Minkowski space $\boldsymbol{L}^{2 n+4}$ with the metric $x \cdot y=x_{1} y_{1}+$ $\cdots+x_{2 n+3} y_{2 n+3}-x_{2 n+4} y_{2 n+4}$. Define $\boldsymbol{X}: M \times \boldsymbol{R} \rightarrow \boldsymbol{H}^{2 n+3} \subset \boldsymbol{L}^{2 n+4}$ by

$$
\boldsymbol{X}\left(\varphi_{1}, \ldots, \varphi_{k}, t\right)=\left(\begin{array}{c}
r(t) \cos \theta(t) \mathbf{m}\left(\varphi_{1}, \ldots, \varphi_{k}\right) \\
r(t) \sin \theta(t) \mathbf{m}\left(\varphi_{1}, \ldots, \varphi_{k}\right) \\
\sqrt{r^{2}+1} \sinh s(t) \\
\sqrt{r^{2}+1} \cosh s(t)
\end{array}\right) .
$$

Then $\boldsymbol{X}$ is a complete minimal immersion, provided $r(t)$ and $\theta(t)$ satisfy

$$
\begin{gathered}
\dot{r}^{2}+\frac{L^{2}}{r^{2}}-r^{2 k}-r^{2 k+2}=-c, \\
r^{2} \dot{\theta}=L
\end{gathered}
$$

and

$$
\dot{s}=\frac{\sqrt{c-L^{2}}}{r^{2}+1},
$$

where $c$ and $L$ are constants.

Proof. Let $d s_{M}^{2}, \bar{g}_{a b}, \bar{g}, \Sigma, d s_{\Sigma}^{2}, g_{a b}, \sqrt{g}$ be the same as in the proof of Theorem 3 . Then

$$
\dot{\boldsymbol{X}}=\left(\begin{array}{c}
(\dot{r} \cos \theta-r \dot{\theta} \sin \theta) \mathbf{m} \\
(\dot{r} \sin \theta+r \dot{\theta} \cos \theta) \mathbf{m} \\
\left(r \dot{r} / \sqrt{r^{2}+1}\right) \sinh s+\sqrt{r^{2}+1} \dot{s} \cosh s \\
\left(r \dot{r} / \sqrt{r^{2}+1}\right) \cosh s+\sqrt{r^{2}+1} \dot{s} \sinh s
\end{array}\right),
$$


and hence by (6), (7), (8),

$$
g_{k+1 k+1}=|\dot{\boldsymbol{X}}|^{2}=\dot{r}^{2}+r^{2} \dot{\theta}^{2}-\frac{r^{2} \dot{r}^{2}}{r^{2}+1}+\left(r^{2}+1\right) \dot{s}^{2}=r^{2 k}
$$

and

$$
g=r^{4 k} \bar{g}
$$

Differentiating (6) and (7) gives

$$
\ddot{r}=\frac{L^{2}}{r^{3}}+k r^{2 k-1}+(k+1) r^{2 k+1}
$$

and

$$
2 \dot{r} \dot{\theta}+r \ddot{\theta}=0 .
$$

Therefore for $i=1, \ldots, n+1$, we have

$$
\begin{aligned}
\Delta_{\Sigma} x_{i} & =\Delta_{\Sigma}\left(r \cos \theta m_{i}\right) \\
& =\frac{1}{r^{2 k} \sqrt{g}}\left[\frac{\partial}{\partial \varphi_{a}}\left\{r^{2 k} \sqrt{\bar{g}} \frac{\bar{g}^{a b}}{r^{2}} \frac{\partial}{\partial \varphi_{b}}\left(r \cos \theta m_{i}\right)\right\}+\frac{\partial}{\partial t}\left\{r^{2 k} \sqrt{\bar{g}} \frac{1}{r^{2 k}} \frac{\partial}{\partial t}\left(r \cos \theta m_{i}\right)\right\}\right] \\
& =\frac{\cos \theta}{r} \frac{1}{\sqrt{g}} \frac{\partial}{\partial \varphi_{a}}\left\{\sqrt{\bar{g}} \bar{g}^{a b} \frac{\partial}{\partial \varphi_{b}} m_{i}\right\}+\frac{1}{r^{2 k}}\left(\ddot{r} \cos \theta-2 \dot{r} \dot{\theta} \sin \theta-r \ddot{\theta} \sin \theta-r \dot{\theta}^{2} \cos \theta\right) m_{i} \\
& =-\frac{k \cos \theta}{r} m_{i}+\left\{\frac{k}{r}+(k+1) r\right\} \cos \theta m_{i} \quad \text { (by (7), (9), (10)) } \\
& =(k+1) x_{i} .
\end{aligned}
$$

Similarly for $i=n+2, \ldots, 2 n+2$, we have

$$
\Delta_{\Sigma} x_{i}=\Delta_{\Sigma}\left(r \sin \theta m_{i}\right)=(k+1) r \sin \theta m_{i}=(k+1) x_{i} .
$$

Now the derivative of $(8)$ is

$$
\ddot{s}=-2 \sqrt{c-L^{2}} \frac{r \dot{r}}{\left(r^{2}+1\right)^{2}} .
$$

Hence

$$
\begin{aligned}
\Delta_{\Sigma} x_{2 n+3}= & \Delta_{\Sigma} \sqrt{r^{2}+1} \sinh s=\frac{1}{r^{2 k} \sqrt{g}} \frac{\partial}{\partial t}\left\{r^{2 k} \sqrt{\bar{g}} \frac{1}{r^{2 k}} \frac{\partial}{\partial t}\left(\sqrt{r^{2}+1} \sinh s\right)\right\} \\
= & \frac{1}{r^{2 k}} \frac{\partial}{\partial t}\left(\frac{r \dot{r}}{\sqrt{r^{2}+1}} \sinh s+\sqrt{r^{2}+1} \dot{s} \cosh s\right) \\
= & \frac{1}{r^{2 k} \sqrt{r^{2}+1}}\left[\left\{\dot{r}^{2}+r \ddot{r}+\left(r^{2}+1\right) \dot{s}^{2}-\frac{r^{2} \dot{r}^{2}}{r^{2}+1}\right\} \sinh s\right. \\
& \left.+\left\{2 r \dot{r} \dot{s}+\left(r^{2}+1\right) \ddot{s}\right\} \cosh s\right] \\
= & (k+1) \sqrt{r^{2}+1} \sinh s \quad(\text { by (9), (11)) } \\
= & (k+1) x_{2 n+3} .
\end{aligned}
$$


Similarly, we have

$$
\Delta_{\Sigma} x_{2 n+4}=(k+1) x_{2 n+4}
$$

THEOREM 5. Let $M$ be a $k$-dimensional complete minimal submanifold of $\boldsymbol{S}^{n}$ locally defined by the immersion $\mathbf{m}\left(\varphi_{1}, \ldots, \varphi_{k}\right)$. Define $\boldsymbol{X}: M \times \boldsymbol{R} \rightarrow \boldsymbol{S}^{2 n+3} \subset \boldsymbol{R}^{2 n+4}$ by

$$
\boldsymbol{X}\left(\varphi_{1}, \ldots, \varphi_{k}, t\right)=\left(\begin{array}{c}
r(t) \cos \theta(t) \mathbf{m}\left(\varphi_{1}, \ldots, \varphi_{k}\right) \\
r(t) \sin \theta(t) \mathbf{m}\left(\varphi_{1}, \ldots, \varphi_{k}\right) \\
\sqrt{1-r^{2}} \cos s(t) \\
\sqrt{1-r^{2}} \sin s(t)
\end{array}\right) .
$$

Then $\boldsymbol{X}$ is a complete minimal immersion, provided $r(t)$ and $\theta(t)$ satisfy

$$
\begin{gathered}
\dot{r}^{2}+\frac{L^{2}}{r^{2}}-r^{2 k}+r^{2 k+2}=-c, \\
r^{2} \dot{\theta}=L
\end{gathered}
$$

and

$$
\dot{s}=\frac{\sqrt{c+L^{2}}}{1-r^{2}}
$$

where $c$ and $L$ are constants for which, if nonzero, (12) has a positive periodic solution $r(t)<1$.

PROOF. The only part of the proof which is different from that of Theorem 4 is the following:

$$
\begin{gathered}
\ddot{r}=\frac{L^{2}}{r^{3}}+k r^{2 k-1}-(k+1) r^{2 k+1}, \\
\ddot{s}=2 \sqrt{c+L^{2}} \frac{r \dot{r}}{\left(1-r^{2}\right)^{2}} .
\end{gathered}
$$

Therefore for $i=1, \ldots, n+1$, we have

$$
\begin{aligned}
\Delta_{\Sigma} x_{i}= & \Delta_{\Sigma}\left(r \cos \theta m_{i}\right) \\
= & \frac{1}{r^{2 k} \sqrt{\bar{g}}}\left[\frac{\partial}{\partial \varphi_{a}}\left\{r^{2 k} \sqrt{\bar{g}} \frac{\bar{g}^{a b}}{r^{2}} \frac{\partial}{\partial \varphi_{b}}\left(r \cos \theta m_{i}\right)\right\}\right. \\
& \left.+\frac{\partial}{\partial t}\left\{r^{2 k} \sqrt{\bar{g}} \frac{1}{r^{2 k}} \frac{\partial}{\partial t}\left(r \cos \theta m_{i}\right)\right\}\right] \\
= & \frac{\cos \theta}{r} \frac{1}{\sqrt{g}} \frac{\partial}{\partial \varphi_{a}}\left\{\sqrt{\bar{g}} \bar{g}^{a b} \frac{\partial}{\partial \varphi_{b}} m_{i}\right\} \\
& +\frac{1}{r^{2 k}}\left(\ddot{r} \cos \theta-2 \dot{r} \dot{\theta} \sin \theta-r \ddot{\theta} \sin \theta-r \dot{\theta}^{2} \cos \theta\right) m_{i} \\
= & -\frac{k \cos \theta}{r} m_{i}+\left\{\frac{k}{r}-(k+1) r\right\} \cos \theta m_{i} \quad \text { (by (13), }
\end{aligned}
$$




$$
=-(k+1) x_{i} .
$$

Finally, we have

$$
\begin{aligned}
\Delta_{\Sigma} x_{2 n+3}= & \Delta_{\Sigma} \sqrt{1-r^{2}} \cos s=\frac{1}{r^{2 k} \sqrt{g}} \frac{\partial}{\partial t}\left\{r^{2 k} \sqrt{\bar{g}} \frac{1}{r^{2 k}} \frac{\partial}{\partial t}\left(\sqrt{1-r^{2}} \cos s\right)\right\} \\
= & \frac{1}{r^{2 k}} \frac{\partial}{\partial t}\left(\frac{-r \dot{r}}{\sqrt{1-r^{2}}} \cos s-\sqrt{1-r^{2}} \dot{s} \sin s\right) \\
= & \frac{1}{r^{2 k} \sqrt{1-r^{2}}}\left[\left\{-\dot{r}^{2}-r \ddot{r}-\left(1-r^{2}\right) \dot{s}^{2}-\frac{r^{2} \dot{r}^{2}}{1-r^{2}}\right\} \cos s\right. \\
& \left.+\left\{2 r \dot{r} \dot{s}-\left(1-r^{2}\right) \ddot{s}\right\} \sin s\right] \\
= & -(k+1) \sqrt{1-r^{2}} \cos s \quad(\text { by (15), (16)) } \\
= & -(k+1) x_{2 n+3} .
\end{aligned}
$$

\section{REFERENCES}

[ 1 ] D. E. Blair, On a generalization of the catenoid, Can. J. Math. 27 (1975), 231-236.

[2] E. Bombieri, E. De Giorgi and E. Giusti, Minimal cones and the Bernstein problem, Invent. Math. 7 (1969), 243-268.

[ 3 ] J. ChoE, On the existence of higher dimensional Enneper's surface, Comment. Math. Helv. 71 (1996), 556569.

[4] C. J. Costa, Example of a complete minimal immersion in $\boldsymbol{R}^{3}$ of genus one and three embedded ends, Bol. Soc. Brasil. Mat. 15 (1984), 47-54.

[ 5 ] J. Simons, Minimal varieties in Riemannian manifolds, Ann. of Math. (2) 88 (1968), 62-105.

KOREA INSTITUTE FOR ADVANCED STUDY

207-43 CHEONGRYANGRI 2-DONG

DONGDAEMUN-GU

SEOUL 130-722

Korea

E-mail address: choe@kias.re.kr

\author{
Department of MATHEMATICS \\ SOGANG UNIVERSITY \\ SHINSU-DONG 1, MAPO-GU \\ SEOUL 121-742 \\ Korea
}

E-mail address: hoppe@sogang.ac.kr 